\title{
ROMANIAN
}

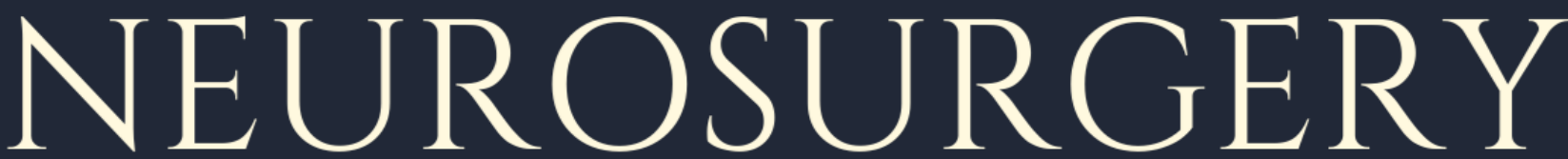

\author{
Vol. XXXIV | No. $1 \quad$ March 2020
}

\section{Mechanical thrombectomy techniques for acute ischemic stroke}

\author{
A. Chiriac, \\ Georgiana Ion, \\ N. Dobrin, \\ Dana Turliuc, \\ I. Poeata
}




\title{
Mechanical thrombectomy techniques for acute ischemic stroke
}

\author{
A. Chiriac, Georgiana Ion ${ }^{1}$, N. Dobrin ${ }^{1}$, Dana Turliuc, I. Poeata \\ 1 "Gr. T. Popa" University of Medicine and Pharmacy, lasi, RomanIA \\ 2 "Prof. Dr. N. Oblu" Clinic Emergency Hospital, Iasi, RomanIA
}

\begin{abstract}
Mechanical thrombectomy technique was introduced as an effective and secure method in acute ischemic stroke patients suffering from intracranial large vessel occlusion (LVO). In this article, we will review the main mechanical thrombectomy techniques and current trends in this type of treatment for acute ischemic stroke.
\end{abstract}

\section{INTRODUCTION}

The large global studies have shown that stroke is the second most common cause of death and third commonest cause of disability in the world. The proven limitations of intravenous and intra-arterial thrombolysis for performing large vascular occlusion recanalization resulted in the introduction of mechanical thrombectomy (MT). This was also favoured by technological advances in endovascular neurosurgery with better catheters, to allow more distal access and more effective devices for efficient intravascular thrombus penetration and capture. Starting with 2015, the role of mechanical thrombectomy for the occlusion of large vessels in acute ischemic stroke has been scientifically proven with the publication of seven randomized controlled trials that demonstrated better outcomes compared to medical management alone. Mechanical thrombectomy evolved from the first generation of thrombus penetration devices, to the direct thrombus-aspiration systems and then to the second generation of devices represented by stent retrievers. In this article we will review the main mechanical thrombectomy techniques and current trends in this type of treatment for acute ischemic stroke [1,2].

The limitations of intravenous thrombolysis have led to the exploration of alternative or complementary treatment approaches for acute ischemic stroke (AIS). Endovascular mechanical thrombectomy has developed over the past years as a safe and efficient intervention for AIS treatment. The fast advancement in catheter and endovascular device technology has led to an increasing number of techniques used in mechanical thrombectomy in patients suffering from an emergent large vessel occlusion.

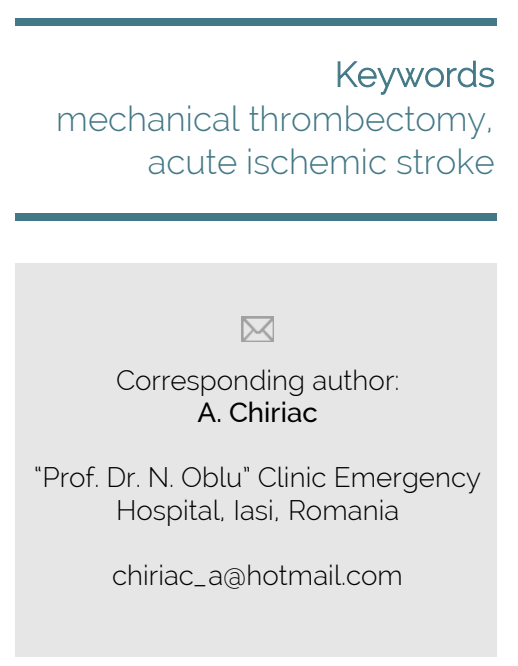

Copyright and usage. This is an Open Access article. distributed under the terms of the Creative Commons Attribution Non-Commercial No Derivatives License (https://creativecommons org/licenses/by-nc-nd/4.0/) which permits noncommercial re-use, distribution, and reproduction in any medium, provided the original work is unaltered and is properly cited.

The written permission of the Romanian Society of Neurosurgery must be obtained for commercial re-use or in order to create a derivative work.

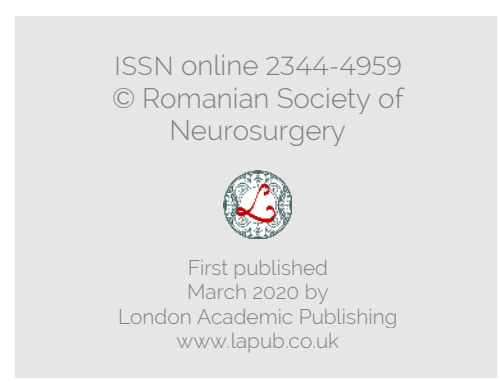




\section{CLOT RETRIEVER TECHNIQUE}

The need to improve the vascular recanalization technique correlated with reducing the risk of cerebral haemorrhage by limiting thrombolytic administration has led to the development of the first mechanical thrombectomy devices. The first steps in the appearance of mechanical thrombectomy devices were performed by Dr. Y. Pierre Gobin of the University of California, Los Angeles, who patented an endovascular device similar to a crib for the recovery of an accidentally released foreign body (usually coils) in the cerebral vasculature. Due to its limited use the device was subsequently taken over and licensed by Concentric Medical, Inc. and called the Mechanical Embolus Removal in Cerebral Ischemia (MERCI) retriever. The Merci retriever is a corkscrew shape device with helical Nitinol loops specifically designed and tested for distal placement and enbloc removal of the thrombus for first time approved by FDA in 2004. This technique involves physical extraction of the thrombus through a catheter (Figure 1). Even though this device has benefited from considerable redesigning in time, all versions were preferably used in conjunction with an 8- or 9-F balloon guiding catheter to reduce the risk of distal emboli $[4,10]$. The target vessels were the proximal segments of major cerebral arteries, predominantly M1 segments of the middle cerebral and vertebrobasilar arteries. MERCI and multi-MERCI trials were prospective, nonrandomized, multicenter, and single-arm trials that reported the safety and efficacy of $\mathrm{MERCl}$ device in patients presenting with moderate to severe stroke (National Institutes of Health Stroke Scale NIHA score $\geq 8$ ) from a large-vessel occlusion and treated within 8 hours of symptom onset[2,4,10].

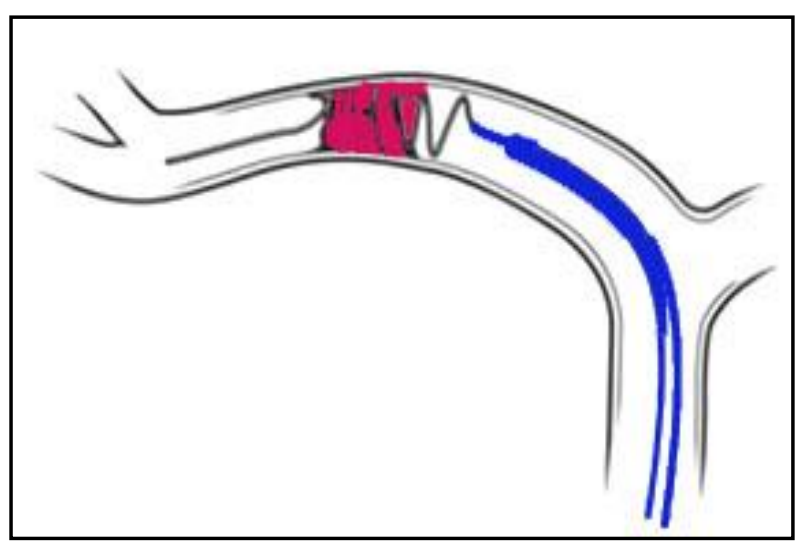

Figure 1: Clot retriever technique

\section{SELF-EXPANDING STENTS TECHNIQUE}

Based of development of the retrieval clot technique, attempts to use self-expanding stents for the purpose of assisting and restoring cerebral revascularization have been reported. Intracranial angioplasty with self-expanding stents was described for the treatment of acute ischemic stroke due to intraarterial atherosclerotic lesions. Even if high rates of stent placement success were achieved immediate and delayed complications were brought into discussion (Figure 2).
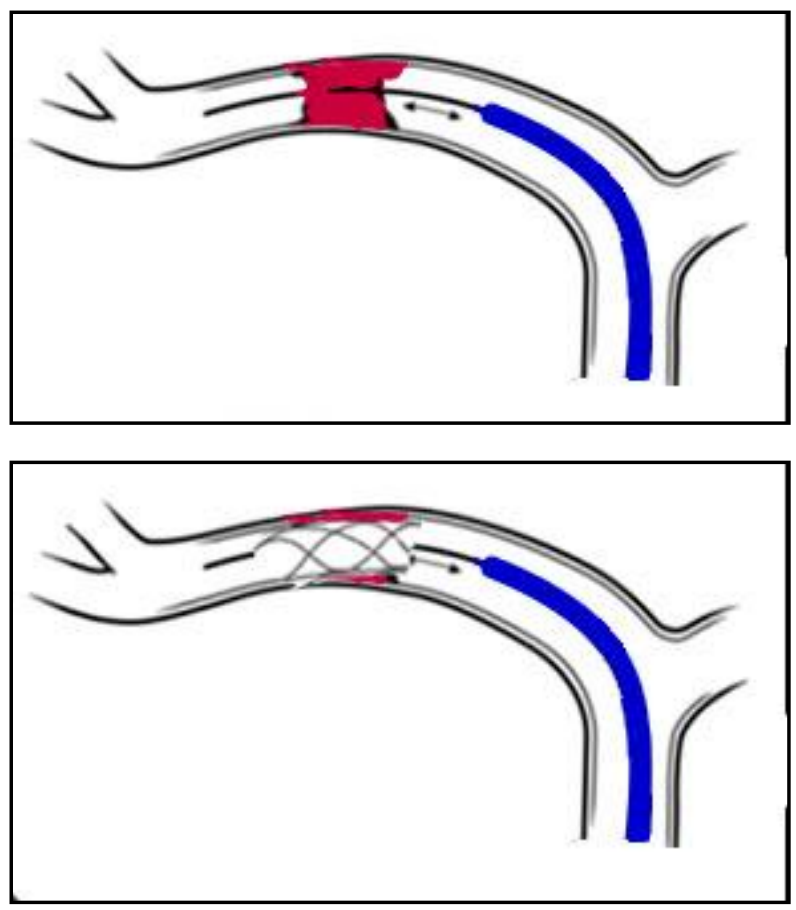

Figure 2: Self-expanding stents technique

Distal emboli due to stent detachment, intra-stent thrombosis or re-stenosis as well as potential haemorrhagic risk of concurrent prophylactic antiplatelet and anticoagulation therapy are just a few of the limitations of the frequent use of this technique. Initial evaluation of the Wingspan stent stent in the SARIS pilot study (ischemic stroke assisted recanalization) in patients with contraindicated or not available intravenous thrombolysis showed a $100 \%$ recanalization rate and a $45 \%$ favorable clinic evolution at 90 days. A $10 \%$ stent related complications was also reported $[3,5]$.

\section{THROMBUS ASPIRATION TECHNIQUE}

The first attempts of thrombectomy by manual suction were described by the use of catheters 
placed in front of the thrombus and by the manual generation of suction through syringes connected to the hub of the catheter, with excellent results $[5,11]$. In December 2007, the Penumbra (PS) system for aspiration of clots (Penumbra, Inc., Alameda, CA) was the second FDA approved device in the treatment of acute ischemic stroke. This technique involves the use of a large lumen catheter device that is advanced through a guide catheter to a point near occlusion, in contact with the clot. In the case of non-adherent clots, with easy mobilization, after capturing them at the top of the suction catheter, it is withdrawn slowly, maintaining the continuous aspiration at a vacuum pump. Simultaneous aspiration is also applied to the lateral port of the intracranial access system (sheath or guide catheter) to prevent thrombus displacement from the tip of the suction catheter, because it is retracted into the sheath. In the case of adherent clots, a microwire called separator is repeatedly passed through the thrombus to disconnect / fragment the clot, and the constant suction is applied to the PS catheter to aspirate the thrombus fragments.

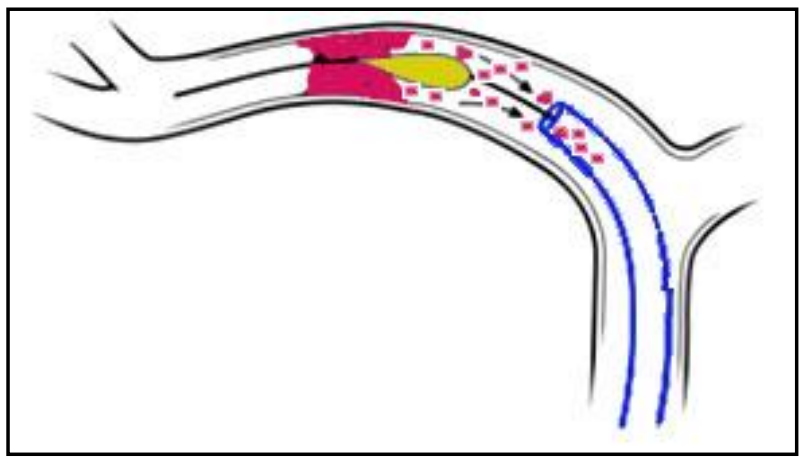

Figure 3: Direct aspiration first pass technique

The introduction of large and flexible suction catheters has led to the development of a new technique called a direct aspiration first pass technique (ADAPT), which can be used as the primary way for revascularization. Kang et al. first reported the use of direct aspiration thrombectomy $[2,5,11]$. In their report, $\mathrm{TICl} 2 \mathrm{~b}-3$ revascularization was performed in $81.9 \%$ of cases. In this technique, the largest possible aspiration catheter is advanced over a microcatheter and microwire (which are used to pass the thrombus and provide stable support for catheter advancement) and positioned immediately adjacent to the occlusion site (Figure 3). Once the suction catheter is in contact with the thrombus, the microcatheter and microwire are removed, and the suction catheter with the clot is withdrawn into the guiding catheter, while continuous negative pressure is applied. Particular attention is given to the moment when the clot is captured, the aspiration forces in the lumen of the vessel are lost and the flow around the intermediate catheter can lead to fragmentation and distal migration during retrieval. This shortcoming can usually be eliminated by using alternating and intermittent aspiration by connecting the continuous aspiration pump tubing into one end of a three-way valve and a $60 \mathrm{cc}$ vacuum syringe. If an optimal control of the catheter advancement exists the aspiration turned on upon contact will be allowed. Thus, the suction force on the thrombus becomes appreciable when the catheter is very close to the thrombus (below $1 \mathrm{~mm}$ ) and may increase until it comes into contact with the thrombus $[2,3,4,5]$.

Another important element that needs to be carefully analyzed is the direction of the suction catheter in relation to the vessel / thrombus to minimize the number of passes and several procedures. In situation of unfavorable angles, a microcatheter over microwire can be used to direct the tip of the aspiration catheter when positioned across the occlusion. The microcatheter/microwire can be kept inside the system during aspiration.

The continuous technological advances allowed the manufacture of last generation of aspiration catheter systems with larger inner-size, excellent navigability, efficacy, and safety profile. Reperfusion catheters such as ACE68, ACE64 and, more recently, JET7 with a suction lumen of 0.072 " (Penumbra) are the best examples. A prospective study initiated by the European Registry on the ACE Reperfusion Catheters and the Penumbra System in the Treatment of Acute Ischemic Stroke (PROMISE, NCT02678169) showed a mTICl 2b-3 revascularization rate of $93.1 \%$, 90-day mRS 0-2 rate of $61 \%$ and a $2.9 \%$ of sICH complication, with $7.5 \%$ mortality at 90-days[13].

Heit J, et al. described in 2018 a so called "SNAKE" technique witch is a technical variation of ADAPT. A SOFIA catheter (Microvention) is advanced into the intracranial circulation without using a guiding wire, microcatheter, or microwire. Because the distal end of the Sofia catheter is extremely soft and pliable it allows a safe "push" of the catheter into different parts of the circle of Willis. When the tip of the Sofia 
is successfully reaches the proximal end of the clot, a manual aspiration with a large syringe can be performed, and the SOFIA catheter is slowly withdrawn into the guide catheter. $[1,2,5]$. Also, the use of smaller thrombo-aspiration catheters such as the Penumbra 3 Max has been reported to be safe and leading to good reperfusion rates especially for distal locations.

\section{STENT RETRIEVER TECHNIQUE}

The stent retrievers represent the second generation of mechanical thrombectomy devices with has derived from nondetachable neurovascular stents used for aneurism coiling support. The introduction of these devices was an important advantage for endovascular treatment of AIS, offering the advantage of navigability and rapid recanalisation of a stent without the potential long-term complications. The detachable Solitaire intracranial stent (Covidien/EV3; Plymouth, MN) turned into a primary clot retriever.

The tehchnique utilize a retrievable stent that is advanced within a microcatheter through the thrombus until a few millimeters distal to the clot. Thus, the stent is deployed capturing the thrombus into the stent struts and displacing it peripherally to the vessel wall thus restoring blood flow. After 3-5 minutes, the microcatheter and stent retriever are simultaneous removed under continuous proximal aspiration with a syringe (Figure 4).

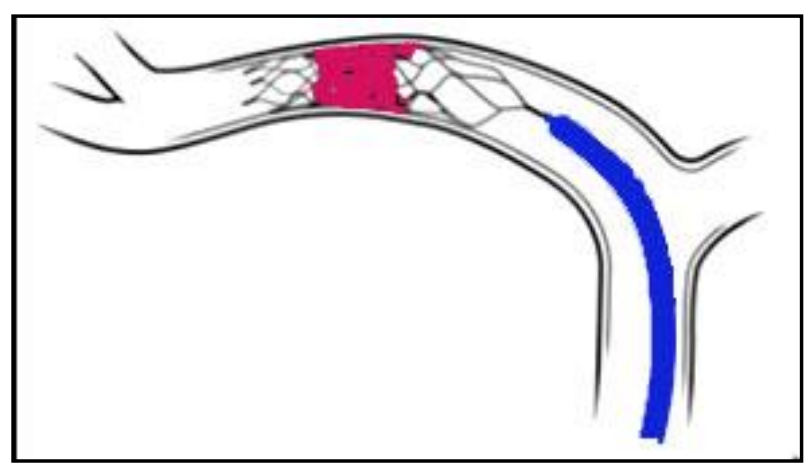

Figure 4: Stent retriever technique

Some improved variants of this technique have also been described in the literature. Haussen et all described an alternative to the conventional unsheathing technique of the stent retriever called Push and Fluff Technique or "active deployment". This particular deployment maneuver consists in positioning the stent retriever across the clot and unsheathing the distal end of the device until good wall apposition (anchoring) is achieved (brief unsheathing step). Then, forward force is applied to the device delivery wire, leading to spontaneous retraction of the microcatheter (pushing step). At the main clot area, additional forward tension is applied to the microcatheter while the delivery wire continues to be pushed to maximize the device struts expansion into embolus (fluffing step). The major concern related to this technique was the potential association with adverse events related to the addition of radial force or forward movement of the device especially in curved vessels. The reports a higher first-pass reperfusion success (modified TICI $2 \mathrm{~b}-3$ with the first pass), $58 \%$ of final full reperfusion and a lower number of overall passes compared to conventional unsheting technique[6].

Dual Stent Retriever Technique was proposed as alternative thrombectomy technique in case of "saddle" bifurcation occlusion. This technique involves the use of two different or identical types of stents retriever that are inserted in parallel or in a $Y$ configuration, with both tips ending in separate bifurcation limbs. The both stent delivery microcatheters require to be placed across the two targeted branches, and then one after another the stent retrievers are deployed at one point. Both stents are gradually retrieved out of the guiding catheter to optimize clot dislodgement. Klisch and colleagues, reports an increased median total recanalization time of 60 minutes (interquartile range 45 to 87 minutes) compared with standard mechanical thrombectomy using single-stent retrieval (range 20 to 48 minutes). Complete recanalization ( $\mathrm{TICl} 2 \mathrm{~b} / 3$ ) was achieved in 8 out of 10 cases, with good clinical outcomes (m Rankin Scale score of 2 or less) in 5 patients[2,7,8].

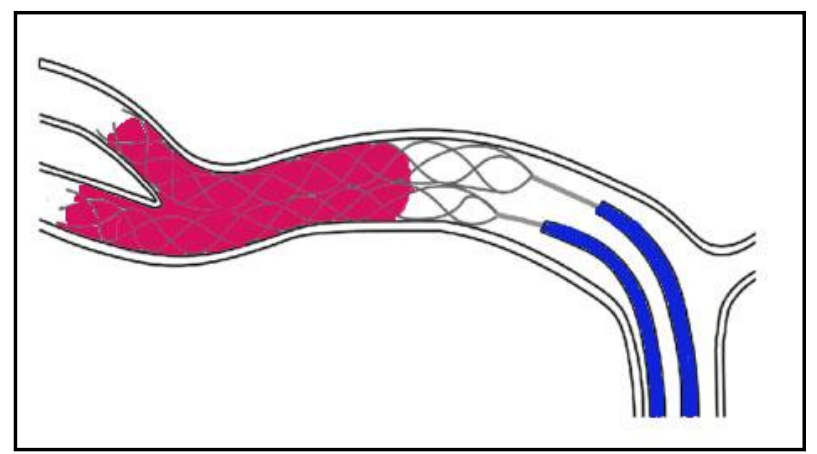

Figure 5: Dual stent retriever technique 


\section{COMBINED TREATMENT}

Aspiration-Retriever Technique also sometime called "Solumbra" due to combination of solitaire stent retrieval and a penumbra aspiration catheter. The technique use a triaxial system which includes an aspiration catheter, a microcatheter and $\mathrm{s}$ microwire. The microcatheter over microwire is advanced through the thrombus and the stent retrieval is deployed across the embolus. Then, the aspiration distal access catheter is advanced close to the proximal end of the stent retrieval in close contact with the thrombus. If necessary the microcatheter can be retracted further to clear the tip of the aspiration catheter optimizing the integration with the thrombus. At the end, the stent retrieval is retracted while continuous negative suction is applied through a pump or manually with an aspiration syringe (Figure 6) $[8,10]$.

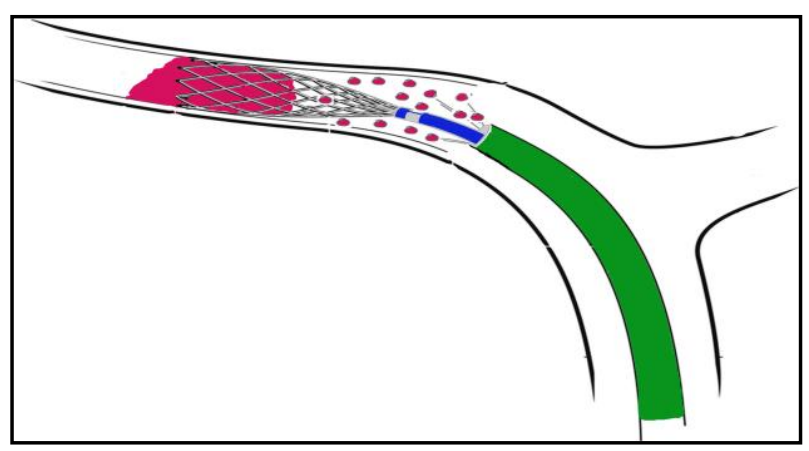

Figure 6: Aspiration-retriever technique

The PROSPECT Technique (proximal balloon occlusion together with direct thrombus aspiration during stent retriever thrombectomy) is combining the use of BGC with large bore distal aspiration catheters that are navigated to the face of the thrombus before retrieving the clot with a stent retriever device. The series of cases published in the literature regarding the use of the PROSPECT technique in the treatment of acute ischemic stroke have shown that the rate of thrombus fragmentations can even be further reduced and the rate of complete and particularly complete first-pass reperfusions can be further increased (Figure 7) $[5,9]$.

The late version of this technique, called PROTECT ${ }^{\text {PLUS }}$ is characterized by the fact that the stent retriever loaded with the clot is not primarily retracted into the aspiration catheter but drawn into the BGC as a unit while aspiration is applied to both the aspiration catheter and the BGC. Maegerlein et all have published in 2018 a study showing that using the PROTECT $^{\text {PLUS }}$ resulted in a higher rate of first pass complete reperfusions ( $59.4 \%$ vs. $27.7 \%, p<0.001$ ) as compared with PROTECT. The PROTECT ${ }^{\text {PLUS }}$ also led to shorter procedure times $(21 \mathrm{~min}$ vs. $37 \mathrm{~min}, p=$ 0.001 ) and higher rates of overall complete reperfusion $(73.5 \%$ vs. $49.5 \%, p=0.014)[5,9,12]$.

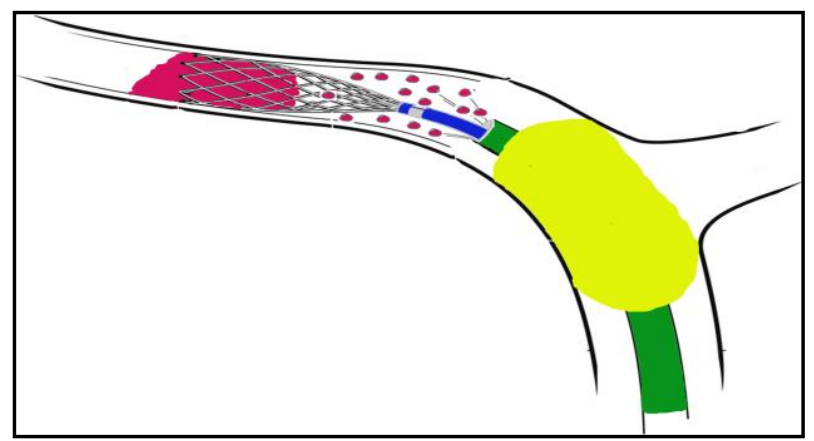

Figure 7: PROSPECT Technique

\section{CONCLUSIONS}

Mechanical thrombectomy approach in large vessel occlusion appears to be a promising method of endovascular stroke treatment, due to a significant increase in the rate of complete first-pass reperfusions and good clinical recovering. Mechanical thrombectomy using the combined techniques seems to be effective, fast and safe with high rates of near-complete and complete reperfusion.

\section{REFERENCES}

1. Alvarez MC., McCarthy DJ., Samir Sur, Snelling BM., Starke M. (2017) Comparing Mechanical Thrombectomy Techniques in the Treatment of Large Vessel Occlusion for Acute Ischemic Stroke, World Neurosurg. Apr;100:681-682.

2. Al-Bayati, A. R., Nogueira, R. G., Samaniego, E. A., \& Haussen, D. C. (2019). Mechanical Thrombectomy: Techniques and Hybrid Approaches for Recanalization. In Acute Stroke Management in the Era of Thrombectomy (pp. 87-103). Springer, Cham.

3. Asadi H, Dowling R, Yan B, Wong S, Mitchell P. Advances in endovascular treatment of acute ischaemic stroke. Intern Med J. 2015;45:798-805.

4. Becker, K. J., \& Brott, T. G. (2005). Approval of the MERCI clot retriever: a critical view. Stroke, 36(2), 400-403.

5. Chueh, J. Y., Kang, D. H., Kim, B. M., \& Gounis, M. J. (2020). Role of Balloon Guide Catheter in Modern Endovascular Thrombectomy. Journal of Korean Neurosurgical Society, 63(1), 14-25. https://doi.org/10.3340/jkns.2019.0114 
6. Haussen, D. C., Rebello, L. C., \& Nogueira, R. G. (2015). Optimizating clot retrieval in acute stroke: the push and fluff technique for closed-cell stentrievers. Stroke, 46(10), 2838-2842.

7. Klisch J, Sychra V, Strasilla C, et al. Double Solitaire mechanical thrombectomy in acute stroke: Effective rescue strategy for refractory artery occlusions? AJNR Am J Neuroradiol 2015;36:552-6.

8. Lapergue, B., Labreuche, J., Blanc, R., Marnat, G., Consoli, A., Rodesch, G., ... \& Duhamel, A. (2020). Combined use of contact aspiration and the stent retriever technique versus stent retriever alone for recanalization in acute cerebral infarction: the randomized ASTER 2 study protocol. Journal of Neurolnterventional Surgery.

9. Maegerlein, C., Berndt, M. T., Mönch, S., Kreiser, K., Boeckh-Behrens, T., Lehm, M., ... \& Friedrich, B. (2018). Further development of combined techniques using stent retrievers, aspiration catheters and BGC. Clinical neuroradiology, 1-7.

10. Massari, F., Henninger, N., Lozano, J. D., Patel, A., Kuhn, A. L., Howk, M., ... \& Wakhloo, A. K. (2016). ARTS (aspirationretriever technique for stroke): initial clinical experience. Interventional Neuroradiology, 22(3), 325-332.

11. Munich, S. A., Vakharia, K., \& Levy, E. I. (2019). Overview of mechanical thrombectomy techniques. Neurosurgery, 85(suppl_1), S60-S67.

12. Puri, A. S., \& Parthasarathy, R. (2019). Aspiration Retriever Technique in Stroke (ARTS). In 100 Interesting Case Studies in Neurointervention: Tips and Tricks (pp. 359362). Springer, Singapore.

13. Schramm, P., Navia, P., Papa, R., Zamarro, J., Tomasello, A., Weber, W., ... \& Gralla, J. (2019). ADAPT technique with ACE68 and ACE64 reperfusion catheters in ischemic stroke treatment: results from the PROMISE study. Journal of neurointerventional surgery, 11(3), 226-231. 